\title{
Thromboembolic and Bleeding Events in COVID-19 Patients receiving Extracorporeal Membrane Oxygenation
}

\author{
Koray Durak ${ }^{1,2,+(0)}$ Alexander Kersten ${ }^{3,+}$ Oliver Grottke ${ }^{4}$ Rashad Zayat $^{10}$ Michael Dreher ${ }^{5}$ \\ Rüdiger Autschbach ${ }^{1}$ Gernot Marx ${ }^{6} \quad$ Nikolaus Marx $^{3} \quad$ Jan Spillner $^{1} \quad$ Sebastian Kalverkamp ${ }^{1}$ \\ ${ }^{1}$ Department of Thoracic and Cardiovascular Surgery, Uniklinik RWTH \\ Aachen, Aachen, Germany \\ 2 Radboudumc, Nijmegen, The Netherlands \\ ${ }^{3}$ Department of Cardiology, Angiology and Intensive Care, Uniklinik \\ RWTH Aachen, Aachen, Germany \\ ${ }^{4}$ Department of Anesthesiology, Uniklinik RWTH Aachen, Aachen, \\ Germany \\ ${ }^{5}$ Department of Pneumology and Intensive Care Medicine, Uniklinik \\ RWTH Aachen, Aachen, Germany \\ ${ }^{6}$ Department of Intensive Care and Intermediate Care Medicine, \\ Uniklinik RWTH Aachen, Aachen, Germany \\ Thorac Cardiovasc Surg 2021;69:526-536. \\ Address for correspondence Koray Durak, Uniklinik RWTH Aachen, \\ Pauwelsstraße 30, Aachen 5207, Germany \\ (e-mail:k.durak@student.ru.nl).
}

\begin{abstract}
Background Extracorporeal membrane oxygenation (ECMO) is a potential treatment option in critically ill COVID-19 patients suffering from acute respiratory distress syndrome (ARDS) if mechanical ventilation (MV) is insufficient; however, thromboembolic and bleeding events (TEBE) during ECMO treatment still need to be investigated. Methods We conducted a retrospective, single-center study including COVID-19 patients treated with ECMO. Additionally, we performed a univariate analysis of 85 preECMO variables to identify factors influencing incidences of thromboembolic events (TEE) and bleeding events (BE), respectively.

Results Seventeen patients were included; the median age was 57 years (interquartile range [IQR]: 51.5-62), 11 patients were males (65\%), median ECMO duration was 16 days (IQR: $10.5-22$ ), and the overall survival was $53 \%$. Twelve patients (71\%) developed TEBE. We observed 7 patients (41\%) who developed TEE and 10 patients (59\%) with BE. Upper respiratory tract (URT) bleeding was the most frequent BE with eight cases (47\%). Regarding TEE, pulmonary artery embolism (PAE) had the highest

Keywords

- COVID-19

- SARS-CoV-2

- ARDS

- ECMO

- thrombosis

- bleeding incidence with five cases (29\%). The comparison of diverse pre-ECMO variables between patients with and without TEBE detected one statistically significant value. The platelet count was significantly lower in the BE group $(n=10)$ than in the non-BE group ( $n=7$ ) with 209 (IQR: $145-238$ ) versus $452 \mathrm{G} / \mathrm{L}$ (IQR: $240-560)$, with $p=0.007$. Conclusion This study describes the incidences of TEE and BE in critically ill COVID-19 patients treated with ECMO. The most common adverse event during ECMO support was bleeding, which occurred at a comparable rate to non-COVID-19 patients treated with ECMO.
\end{abstract}

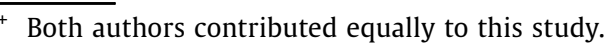

received

November 28, 2020

accepted after revision

January 18, 2021

published online

April 16, 2021 (c) 2021. Thieme. All rights reserved.

Georg Thieme Verlag KG,

Rüdigerstraße 14,

70469 Stuttgart, Germany
DOI https://doi.org/ 10.1055/s-0041-1725180. ISSN $0171-6425$. 


\section{Introduction}

The severe acute respiratory syndrome coronavirus 2 (SARSCoV-2) already caused over 10 million infections and 500,000 deaths as reported in July $2020{ }^{1}$ SARS-CoV-2 infection causes hyperactivity of immune and nervous systems and an activation of the coagulation cascade associated with severe hypercoagulability, leading to multiple organ failure. ${ }^{2}$ The hypercoagulability leads to an increased risk of venous and arterial thromboembolism with a reported incidence of $31 \%$ for intensive care unit (ICU) patients with COVID-19., ${ }^{3,4}$ Furthermore, COVID-19 acute respiratory distress syndrome (ARDS) patients developed significantly more thrombotic complications than non-COVID-19 ARDS patients (11.7 vs. $2.1 \%$, $p<0.008) .{ }^{5}$ Certain laboratory parameters, such as D-dimer and fibrin degradation products (FDP), have been shown to identify patients at risk of thromboembolic and bleeding events (TEBE). ${ }^{2,6,7}$ Thereupon studies investigated different regimes for prophylactic anticoagulant therapy in COVID-19 patients at risk of TEBE. The use of heparin is associated with a significantly lower mortality and better prognosis. ${ }^{8}$

Extracorporeal membrane oxygenation (ECMO) plays a role as a rescue therapy. ${ }^{9,10}$ In severe non-COVID-19-induced ARDS patients, there is existing conclusive evidence linking (venovenous [V-V]) ECMO support to better patient outcomes and decreased mortality. ${ }^{11-13}$ However, ECMO treatment itself increases the risk of TEBE ${ }^{14}$ and experience of ECMO treatment in COVID-19 patients remains limited. ${ }^{9,15-17}$

The aim of this study was to describe TEBE in critically ill COVID-19 patients receiving ECMO support. Furthermore, we aimed to identify pre-ECMO characteristics that influence the incidence of TEBE in COVID-19 patients treated with ECMO.

\section{Materials and Methods}

\section{Patients and COVID-19 Diagnosis}

This single-center retrospective observational study included all adult inpatients ( $\geq 18$ years) between March 1 and April 20, 2020, who were diagnosed with COVID-19 according to the WHO interim guidance ${ }^{10}$ and developed severe COVID-19 disease with ARDS requiring ECMO support. Severe cases of COVID-19 with ARDS were identified according to the Berlin Definition of the European Society of Intensive Care Medicine from 2011.

The study was approved by the local ethic commission and the requirement for informed consent was waived by the ethical board due to emerging worldwide public health crisis and retrospective nature of the study.

\section{Data Collection}

Beside demographics, medical history, and treatment regimes, time course of laboratory, mechanical ventilation (MV) parameters, and ECMO settings were collected through the entire length of hospital stay (LOS) and outcomes were extracted from our patient data management systems (Philips IntelliSpace Critical Care and Anesthesia and Siemens Medico). Outcomes comprised mortality and complications such as TEBE, organ failure, super infections, and other pathologies. We also gathered data about the amount of packed red blood cells (PRBC) and albumin units. PRBC units contained 200 to $300 \mathrm{~mL}$ and albumin units were $100 \mathrm{~mL}$ with a concentration of $20 \%$.

\section{Laboratory Analysis}

Detailed laboratory analyses were performed daily and included complete blood count electrolytes; measures of hemostasis; hemolysis markers; biochemical tests of cardiac, renal, and liver function; NT-pro-brain natriuretic peptide (NT-proBNP); interleukin-6 (IL-6); procalcitonin (PCT); C-reactive protein (CRP); fibrinogen; and D-dimers. Blood gas analyses were performed in intervals of 1 to 2 hours. The data were collected afterward, and some laboratory parameters were not always documented in the system over the entire ICU stay. However, the pre-ECMO documentation was complete and missing values exist only in measurements after ECMO initiation.

\section{Extracorporeal Membrane Oxygenation Settings}

Critically ill COVID-19 patients, who presented with commonly accepted ECMO indications as suggested by the Extracorporeal Life Support Organization (ELSO), ${ }^{18}$ in whom all other treatments options have been exhausted (lung protective invasive MV; prone positioning; neuromuscular blockade; and inhaled nitric oxide [iNO] rescue therapy) were considered for ECMO treatment. Decision on initiation of ECMO treatment was judged by consensus following discussion in the ECMO team consisting of internal medicine intensivist, cardiothoracic surgeon, and pneumologist.

The type of ECMO engines were iLA ACTIVVE XLUNG kits (XENIOS, Heilbronn, Germany) and Cardiohelp HLS systems Version 7.0 (Maquet Cardiopulmonary GmbH, Rastatt, Germany). Data about ECMO settings, utilization (i.e., V-V or veno-arterial [V-A]), cannulation sites, utilization switch (i.e., from V-V to V-A or V-VA), and details on ECMO's weaning were extracted. Our standard approach for the treatment of isolated respiratory failure is V-V ECMO.

\section{Anticoagulation Regime}

Hemostasis parameters were measured daily and included activated partial thromboplastin time (aPTT), international normalized ratio (INR), platelet count, fibrinogen, antithrombin, d-dimer, and activated clotting time (ACT). aPTT was measured three times daily and ACT was measured four times daily as a control for adequate coagulation and not as a target value. In addition, factor XIII was measured three times a week. Although these measurements were performed and controlled regularly, the documentation in the system was not complete for all variables.

In all patients with VV-ECMO, we primarily administered $400 \mathrm{IE} / \mathrm{h}$ of unfractionated heparin (UFH) if no other relevant indications for a higher anticoagulation target, like atrial fibrillation or mechanical heart valve prosthesis, were present. If no bleeding complications occurred, we tolerated an ACT up to 180 seconds. If necessary, UFH was reduced or paused. Other target values were an aPTT between 40 and under 60 seconds, platelet count above $50 \mathrm{G} / \mathrm{L}$, and fibrinogen above $150 \mathrm{mg} / \mathrm{dL}$, and we kept the INR under 1.5 . In case 
of bleedings, we adjusted the target values by using fresh frozen plasma or PRBC. We reduced the ACT under 160 seconds, normalized the INR, and raised the platelet count to at least $80 \mathrm{G} / \mathrm{L}$ and fibrinogen to more than $200 \mathrm{mg} / \mathrm{dL}$.

\section{Thromboembolic and Bleeding Events}

TEBE include all thromboembolic events (TEE) and bleeding events (BE) that occurred during ECMO therapy. TEE comprised pulmonary artery embolism (PAE), venous or arterial thrombosis, and ischemic or hemorrhagic stroke. For BE, we documented upper respiratory tract (URT) bleedings and divided them into endobronchial and mucosal bleedings. We also reported the incidence of pericardial tamponade, hemothorax, and cannulation side or gastrointestinal bleedings (GIB).

Screening of the above-mentioned complications was conducted daily due to our standard clinical documentation. BE were differentiated into major and minor in case of URT or cannulation side bleedings. Therefore, all bleedings that led to the use of two or more units of whole blood or red blood cells and/or a fall in hemoglobin more than $1.24 \mathrm{mmol} / \mathrm{L}$ were identified as major according to the definition of a major bleeding from the International Society on Thrombosis and Haemostasis (ISTH). ${ }^{19}$ We chose the ISTH classification due to its applicability for patients treated with anticoagulants and ECMO being a nonsurgical treatment.

Transfusion of PRBC was very frequent and in most patients, we aimed to maintain a hemoglobin value of $9 \mathrm{~g} /$ dL. However, as our main ECMO target aside from ultraprotective ventilation would be a sufficient oxygen delivery, we calculated the ratio of oxygen delivery $\left(\mathrm{Do}_{2}\right)$ to oxygen consumption $\left(\mathrm{Vo}_{2}\right)$ several times daily and aimed for a ratio of $\geq 4: 1$ or at least $3: 1$. Thus, in some patients, a transfusion of PRBC was required due to low $\mathrm{Do}_{2}: \mathrm{Vo}_{2}$ ratios even if hemoglobin was around $10 \mathrm{~g} / \mathrm{dL}$.

\section{Confirmation of SARS-CoV-2}

Throat-swab specimens, tracheal secretions, or bronchoscopic alveolar lavage were obtained for SARS-CoV-2 from each suspected patient immediately at hospital admission. The real-time reverse transcription polymerase chain reaction (RT-PCR) assay was used to confirm COVID-19 infections.

\section{Statistical Analysis}

Categorical variables are presented as absolute numbers $(n)$ and percentages (\%). Continuous variables were tested for normal distribution with the Kolmogorov-Smirnov test and presented as median and interquartile range (IQR).

For comparison between patients with and without TEE or $\mathrm{BE}$, univariate analyses were performed using the MannWhitney $U$ test for continuous variables and Fisher's exact test for categorical variables.

All statistical comparisons were two-sided. A $p$ value $<0.05$ was considered significant. Statistical analysis was performed with SPSS Version 26 (IBM Corp., Armonk, New York, United States). The figure for time courses of laboratory parameters using mean values was created with Microsoft Excel for Mac, Version 16.41 (Microsoft, Redmond, Washington, United States).

\section{Results}

\section{Baseline Characteristics}

We included 17 patients with COVID-19 who received ECMO from March 1 to April 20, 2020. The median age was 57 years (IQR: 52-62), median BMI was $28 \mathrm{~kg} / \mathrm{m}^{2}$ (IQR: 25-32), 6 patients (35\%) were female, and the most prevalent comorbidity was arterial hypertension in 13 patients (76\%). Nine patients (53\%) had fever at admission and the most prevalent typical COVID-19 symptom was dyspnea with 12 patients (71\%). Four patients (24\%) were nicotine users, and 3 patients (18\%) were suffering from COPD. Data on MV and laboratory tests prior to receiving ECMO showed a median $\mathrm{PaO}_{2} / \mathrm{FiO}_{2}$ ratio of 0.82 (IQR: $0.68-1.04$ ) and the median duration of MV before ECMO was 3 days (IQR: 2.5-17). Eight patients (47\%) had iNO inhalation prior to ECMO support and all patients received antibiotic and prone position treatment. We found a median $\mathrm{PaO}_{2}$ of $68 \mathrm{~mm} \mathrm{Hg}$ (IQR: 54-74), $\mathrm{PaCO}_{2}$ of $66 \mathrm{~mm} \mathrm{Hg}$ (IQR: $45-78$ ), d-dimer of $4547 \mu \mathrm{g} / \mathrm{dL}$ (IQR: 1,720-15,395), and fibrinogen of $656 \mathrm{mg} / \mathrm{dL}$ (IQR: 421-717). Furthermore, we found a median platelet value of 226 (IQR: 189-421) and the median PTT was 33 seconds (IQR: 28-37). The Respiratory ECMO Survival Prediction (RESP) score was estimated before ECMO therapy and predicted a median score of -1 (IQR: -3 to 1 ). Further parameters and details are presented in -Table 1.

During ECMO therapy, we observed TEE in 7 patients and BE in 10 patients. We compared the TEE group $(n=7)$ with a non-TEE group $(n=10)$, and the BE group $(n=10)$ with a non-BE group $(n=7)$. Besides patient characteristics, we included laboratory measurements, MV parameters, ECMO settings, and ICU risk scores before ECMO implantation. Baseline characteristics of all groups are provided in - Table 1.

In the first comparison between the TEE group and the non-TEE group, we found no statistically significant differences. However, there were several notable differences. In the seven patients with TEE, we found fewer female patients (14 vs. $50 \%, p=0.160$ ), more patients suffering from atrial fibrillation (57 vs. 20\%, $p=0.162$ ), and less utilization of cytokine absorption ( 29 vs. $60 \%, p=0.335$ ). Furthermore, the TEE group had higher median $\mathrm{pCO}_{2}$ values with 77 (IQR: $55-$ 80 ) versus 60 (IQR: $34-70$ ), with $p=0.088$; and the group also had lower median RESP score values with -2 (IQR: -4 to 0 ) versus 0 ( -3 to 2 ), $p=0.315$.

As for the 10 patients with $\mathrm{BE}$, we found one statistically significant difference and some notable differences regarding coagulation parameters. The median platelet count was significantly lower in the BE group than in the non-BE group with 209 (IQR: 145-238) versus 452 (IQR: 240-560), $p=0.007$. The BE group also had a longer median PTT with 35 (IQR: 28-39) versus 29 (IQRL 28-34) seconds, $p=0.193$; and the group also had a lower median INR with 1.2 (IQR: $1.2-1.3$ ) versus 1.3 (IQR: $1.3-1.4$ ), $p=0.133$. 


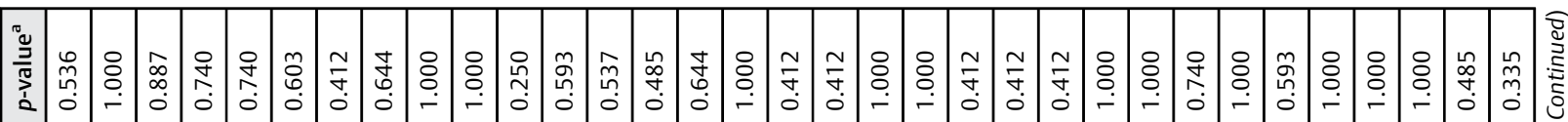

玄高

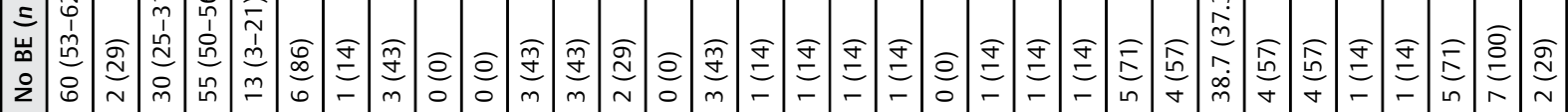

ลุ.

$m$

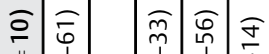

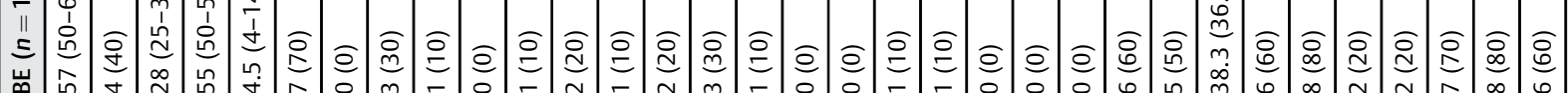

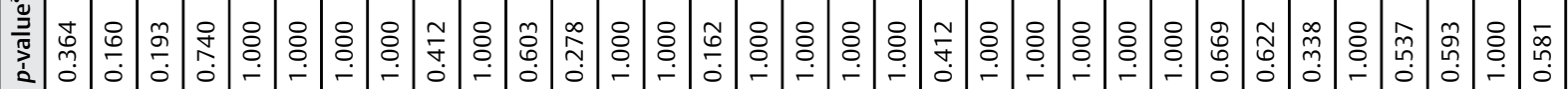

은

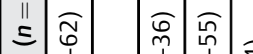

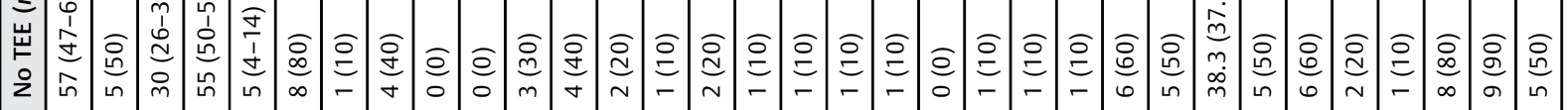

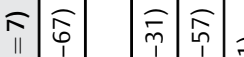

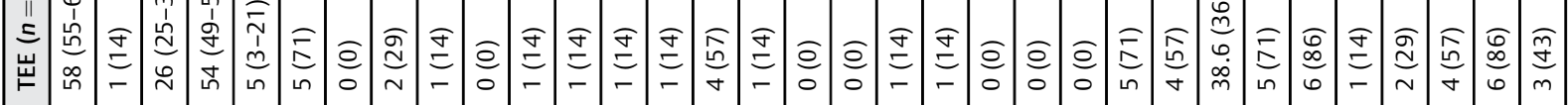

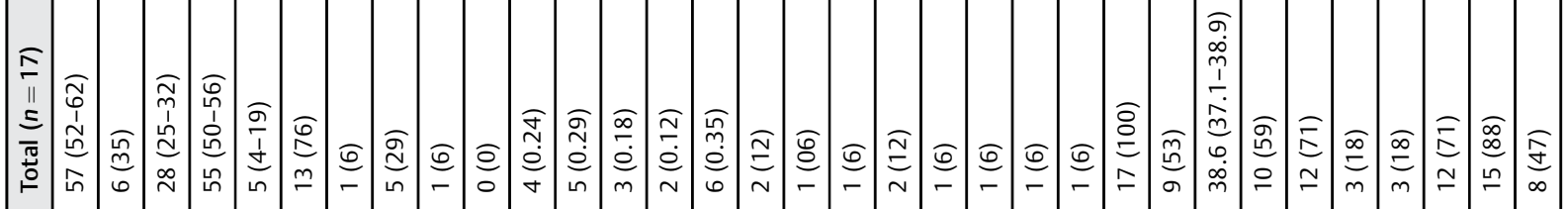

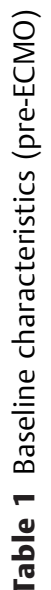

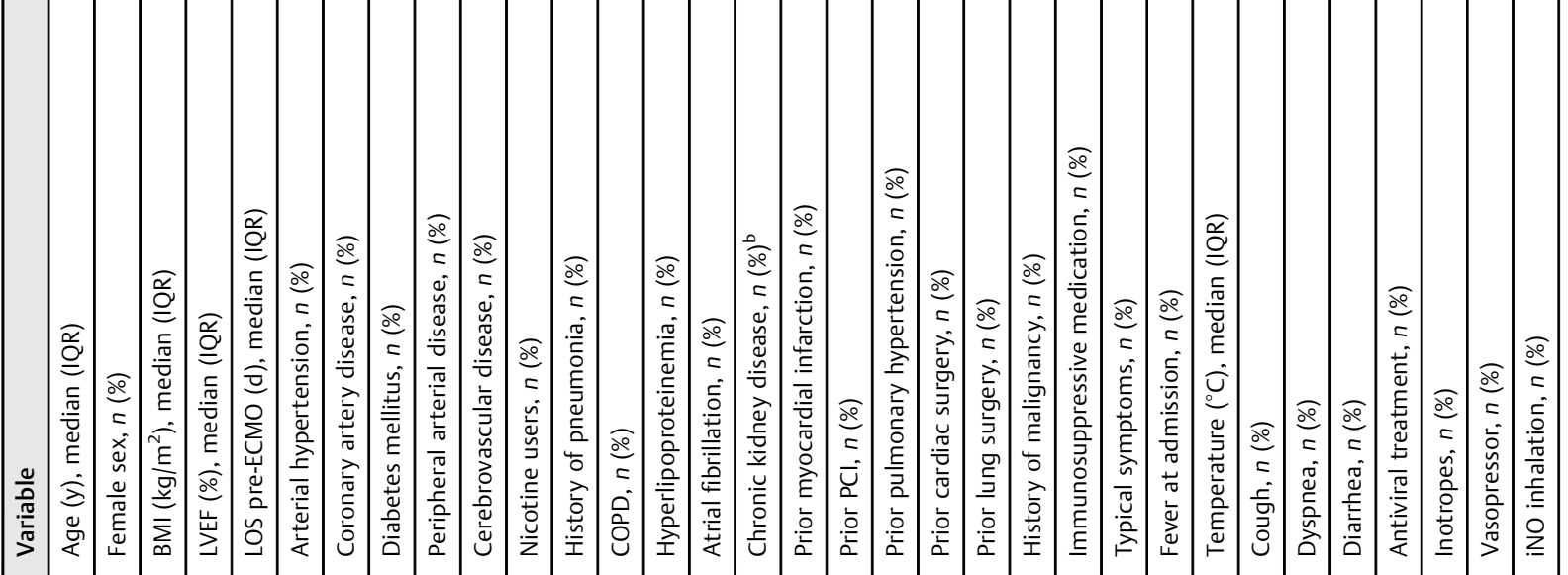




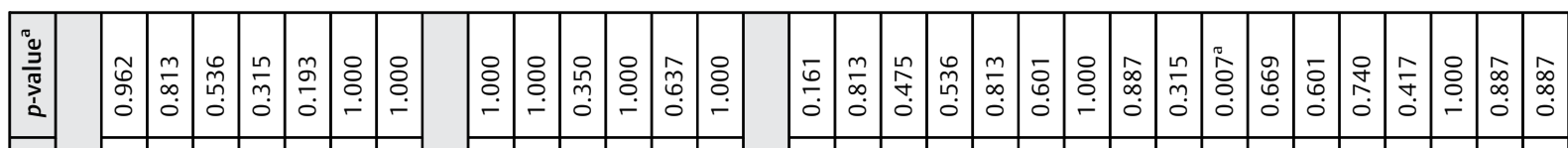

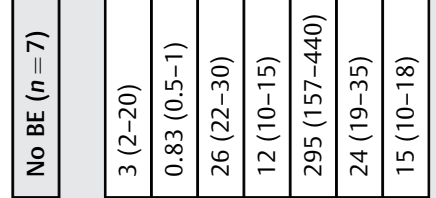

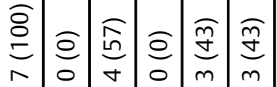

官

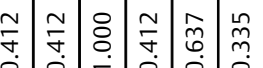

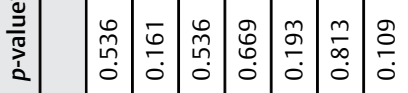

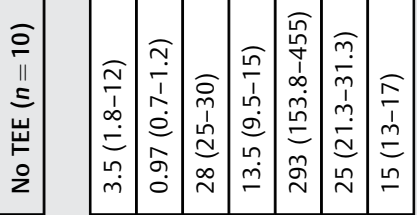

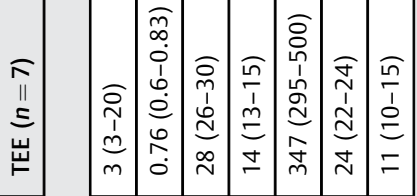

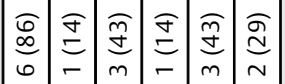

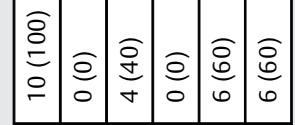

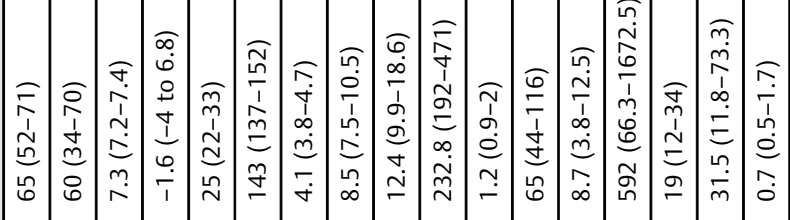

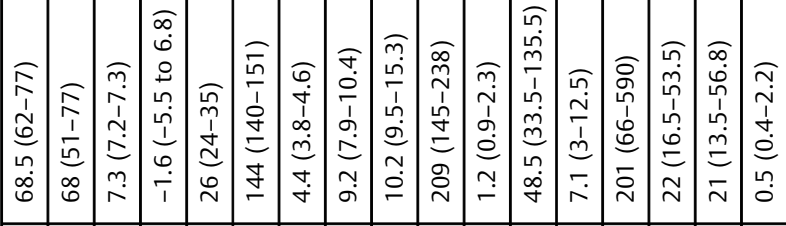

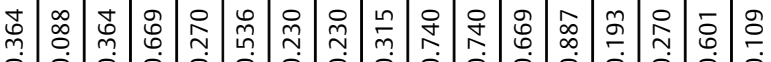

$\begin{array}{lllllllllllllllllllllllll}0 & 0 & 0 & 0 & 0 & 0 & 0 & 0 & 0 & 0 & 0 & 0 & 0 & 0 & 0 & 0 & 0\end{array}$ 


\begin{tabular}{|c|c|c|c|c|c|c|c|c|c|c|c|c|c|c|c|c|c|c|c|c|c|c|}
\hline$\frac{\text { 프 }}{\pi}$ & 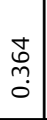 & {$\left[\begin{array}{c}\infty \\
\infty \\
0 \\
0 \\
0\end{array}\right]$} & 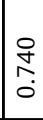 & $\mid \begin{array}{c}0 \\
\infty \\
0 \\
0 \\
0\end{array}$ & $\frac{\tilde{2}}{0}$ & $\begin{array}{c}m \\
\stackrel{m}{0} \\
\end{array}$ & 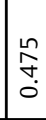 & $\mid \begin{array}{l}m \\
\infty \\
0 \\
0\end{array}$ & $\mid \begin{array}{l}0 \\
\tilde{n} \\
0 \\
0\end{array}$ & $\begin{array}{l}\frac{9}{0} \\
\vdots \\
0\end{array}$ & $\begin{array}{l}0 \\
0 \\
0 \\
0 \\
0\end{array}$ & \begin{tabular}{|l}
9 \\
$\hat{T}$ \\
0 \\
0
\end{tabular} & $\mid$\begin{tabular}{|c|}
0 \\
$\infty$ \\
0 \\
0
\end{tabular} & \begin{tabular}{|c|c}
$m$ \\
0 \\
0 \\
0 \\
0
\end{tabular} & \begin{tabular}{l}
9 \\
\multirow{2}{A}{} \\
0 \\
0
\end{tabular} & $\begin{array}{l}\bar{\sigma} \\
\dot{0} \\
\dot{0}\end{array}$ & $\begin{array}{c}\frac{1}{m} \\
\dot{m} \\
\vdots\end{array}$ & \begin{tabular}{|c|c}
0 \\
$\infty$ \\
0 \\
0
\end{tabular} & $\begin{array}{l}9 \\
\hat{T} \\
0 \\
0\end{array}$ & $\mid \begin{array}{l}0 \\
\delta \\
0 \\
0\end{array}$ & \begin{tabular}{|l}
0 \\
$\tilde{n}$ \\
0 \\
0
\end{tabular} & \begin{tabular}{|l}
0 \\
$\delta$ \\
0 \\
0
\end{tabular} \\
\hline $\begin{array}{l}\| \\
\Xi\end{array}$ & 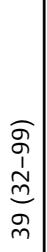 & 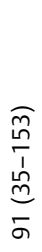 & 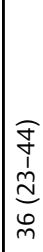 & $\mid \begin{array}{c}\tilde{\sigma} \\
\hat{0} \\
1 \\
\tilde{N} \\
\tilde{n} \\
\tilde{\sigma} \\
\tilde{q}\end{array}$ & $\mid \begin{array}{l}\tilde{f} \\
\tilde{1} \\
\infty \\
\stackrel{0}{0} \\
\stackrel{2}{N}\end{array}$ & 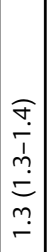 & 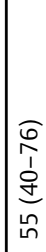 & 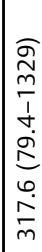 & 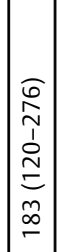 & $\begin{array}{c}0 \\
0 \\
0 \\
1 \\
\dot{1} \\
e \\
\\
\tilde{n} \\
\dot{n}\end{array}$ & 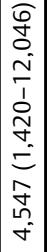 & 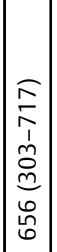 & 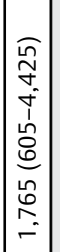 & $\mid \begin{array}{l}\widetilde{T} \\
0 \\
\tilde{I} \\
\underline{1} \\
\tau \\
1\end{array}$ & $\mid \begin{array}{l}\widehat{m} \\
\tilde{m} \\
m\end{array}$ & 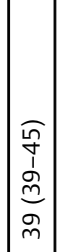 & 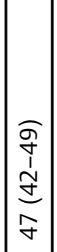 & 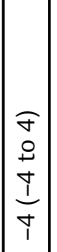 & 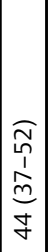 & $\begin{array}{c}\widehat{m} \\
\stackrel{1}{c} \\
\stackrel{n}{n} \\
\stackrel{n}{v}\end{array}$ & 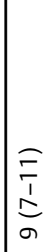 & 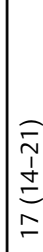 \\
\hline 이 & 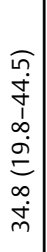 & 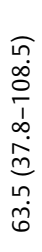 & 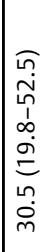 & 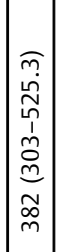 & 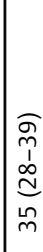 & 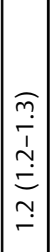 & 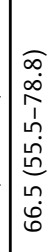 & 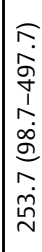 & 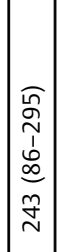 & 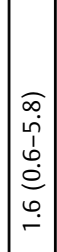 & 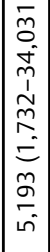 & 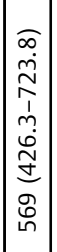 & 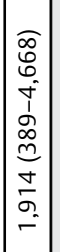 & $\begin{array}{l}0 \\
0 \\
0 \\
n n \\
\hat{1} \\
1 \\
\omega n \\
0 \\
i\end{array}$ & $\begin{array}{l}\widehat{m} \\
\dot{m} \\
m\end{array}$ & 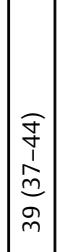 & 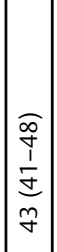 & 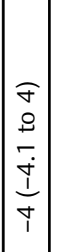 & 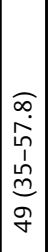 & $\begin{array}{c} \\
\tilde{m} \\
\stackrel{p}{1} \\
\stackrel{d}{c} \\
\sim \\
\sim\end{array}$ & $\begin{array}{l}0 \\
0 \\
\dot{n} \\
1 \\
\Sigma \\
= \\
=\end{array}$ & 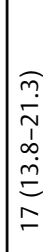 \\
\hline$\frac{\mathrm{g}}{\overline{0}}$ & 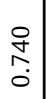 & 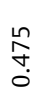 & $\mid \begin{array}{c}0 \\
\infty \\
\infty \\
0\end{array}$ & $\left|\begin{array}{c}m \\
\dot{\alpha} \\
\dot{\alpha}\end{array}\right|$ & 辛 & $\mid \begin{array}{l}1 \\
\infty \\
\infty \\
0 \\
0\end{array}$ & 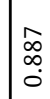 & 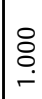 & $\left|\begin{array}{c}m \\
\infty \\
0 \\
0\end{array}\right|$ & $\mid \begin{array}{c}0 \\
\tilde{ָ} \\
0\end{array}$ & $\mid \begin{array}{l}\tilde{n} \\
\hat{f} \\
0\end{array}$ & $\left|\begin{array}{l}0 \\
0 \\
0 \\
0\end{array}\right|$ & $\mid \begin{array}{l}\stackrel{\theta}{ } \\
\stackrel{-}{-}\end{array}$ & $\left|\begin{array}{c}\frac{n}{m} \\
m \\
0\end{array}\right|$ & $\mid \begin{array}{l}0 \\
0 \\
0 \\
0\end{array}$ & $\left|\begin{array}{c}n \\
\hat{f} \\
0 \\
0\end{array}\right|$ & 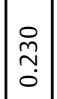 & $\mid \begin{array}{l}m \\
\frac{2}{0} \\
0\end{array}$ & $\mid \begin{array}{l}n \\
\hat{f} \\
0\end{array}$ & $\mid \begin{array}{l}0 \\
8 \\
0\end{array}$ & $\mid \begin{array}{l}0 \\
0 \\
0 \\
0\end{array}$ & $\mid \begin{array}{l}\mathscr{0} \\
\tilde{n} \\
0\end{array}$ \\
\hline o & 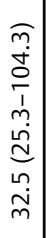 & 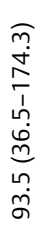 & 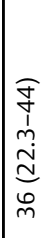 & $\mid$\begin{tabular}{|c}
$\widehat{\sigma}$ \\
0 \\
1 \\
$\hat{0}$ \\
0 \\
0 \\
$o$ \\
$\tilde{m}$ \\
\end{tabular} & 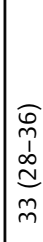 & 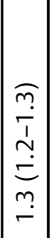 & $\begin{array}{l}0 \\
0 \\
1 \\
0 \\
0 \\
0 \\
0 \\
0 \\
0\end{array}$ & 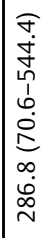 & 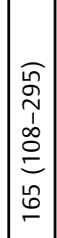 & 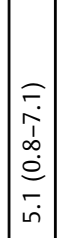 & 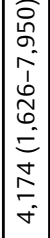 & 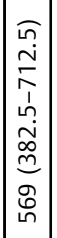 & 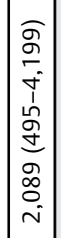 & $\left|\begin{array}{l}\widehat{v} \\
0 \\
0 \\
\underline{1} \\
0\end{array}\right|$ & $\begin{array}{l}\widehat{c} \\
\tilde{m} \\
m\end{array}$ & 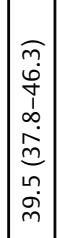 & 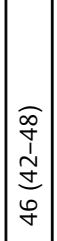 & 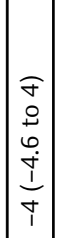 & 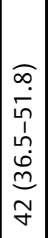 & 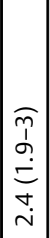 & 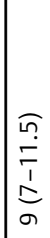 & 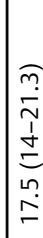 \\
\hline 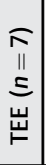 & 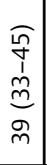 & $\begin{array}{l}\widehat{\overline{9}} \\
\frac{1}{0} \\
0 \\
0 \\
0\end{array}$ & $\begin{array}{l}\widehat{o} \\
0 \\
\vdots \\
d \\
\\
\end{array}$ & $\left|\begin{array}{l}\frac{1}{d} \\
\underline{m} \\
\tilde{g} \\
\dot{\sigma}\end{array}\right|$ & 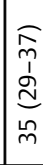 & 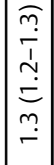 & $\begin{array}{l}\widehat{\widehat{T}} \\
0 \\
1 \\
1 \\
1 \\
0 \\
0 \\
0\end{array}$ & 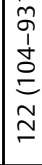 & 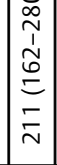 & 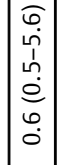 & $\begin{array}{l}0 \\
0 \\
0 \\
= \\
= \\
0 \\
0 \\
\llcorner \\
= \\
= \\
=\end{array}$ & 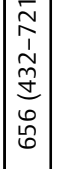 & 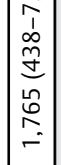 & $\mid \begin{array}{l}0 \\
0 \\
0 \\
y \\
\dot{1} \\
\mathcal{1} \\
1\end{array}$ & $\mid \begin{array}{l}\widehat{m} \\
\stackrel{m}{m} \\
m\end{array}$ & 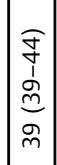 & 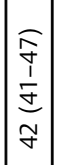 & 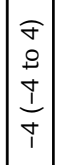 & 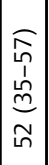 & 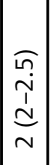 & $\begin{array}{l}\widehat{m} \\
\frac{1}{1} \\
\Sigma \\
=\end{array}$ & 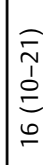 \\
\hline 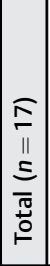 & 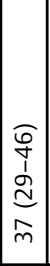 & $\begin{array}{l}\frac{\sigma}{\sigma} \\
\overline{1} \\
\infty \\
0 \\
0 \\
0 \\
0\end{array}$ & $\begin{array}{l}\frac{1}{d} \\
\stackrel{d}{0}\end{array}$ & $\left|\begin{array}{c}n \\
1 \\
n \\
o \\
o \\
0 \\
m \\
\tilde{g}\end{array}\right|$ & $\begin{array}{l}\widehat{\hat{m}} \\
\infty \\
\stackrel{\infty}{n} \\
\tilde{m} \\
\tilde{m}\end{array}$ & 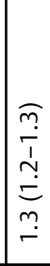 & 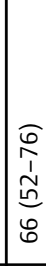 & 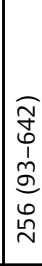 & 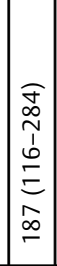 & 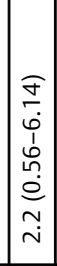 & 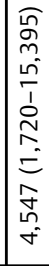 & 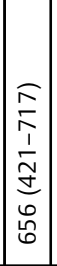 & 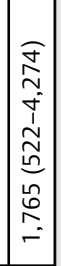 & $\mid \begin{array}{l}0 \\
0 \\
0 \\
m \\
1 \\
1\end{array}$ & $\begin{array}{l}\bar{m} \\
m \\
m \\
m\end{array}$ & 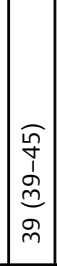 & 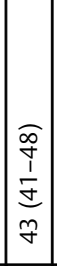 & 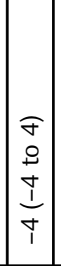 & 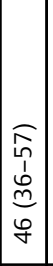 & $\begin{array}{c} \\
\tilde{n} \\
\stackrel{1}{\sim} \\
\stackrel{\sim}{\sim}\end{array}$ & 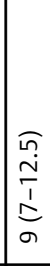 & 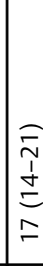 \\
\hline
\end{tabular}

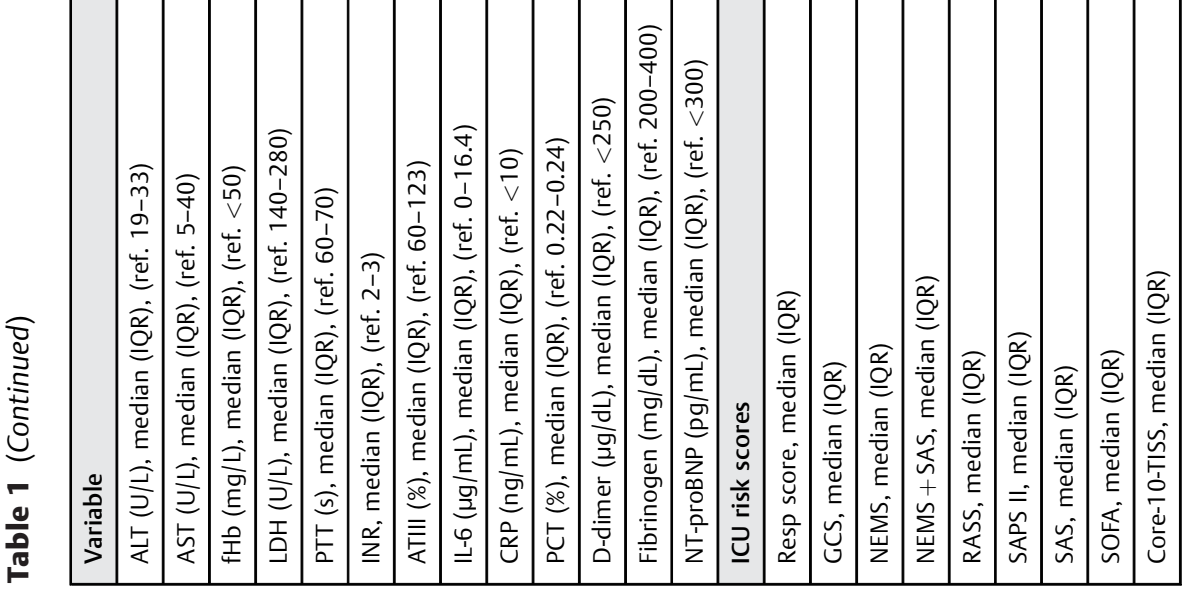

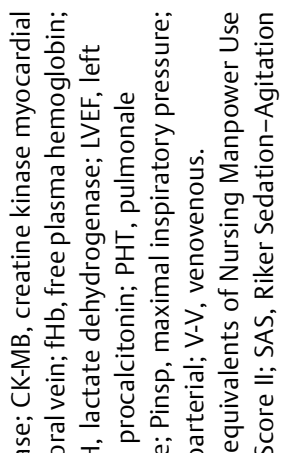

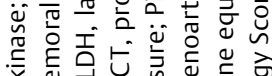

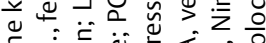

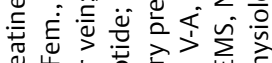

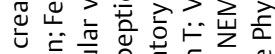

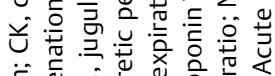

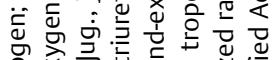

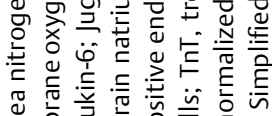

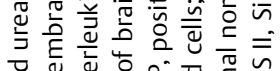
论 응 든 خ்

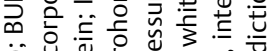

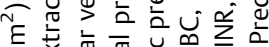

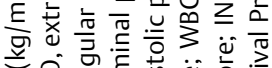

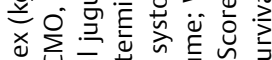

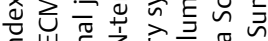

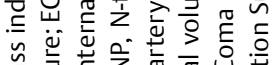

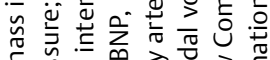

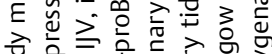
중 는 준

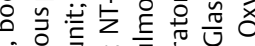

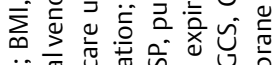

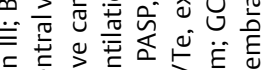

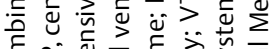

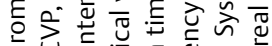

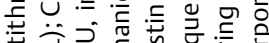

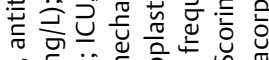

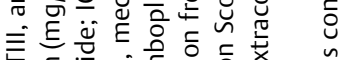

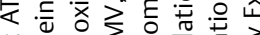

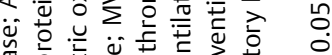

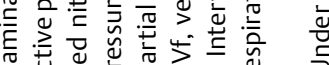

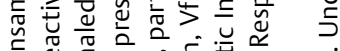

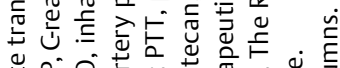

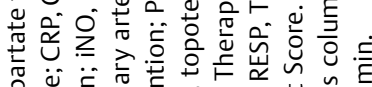

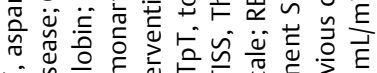

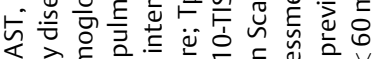

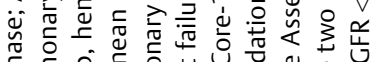
.

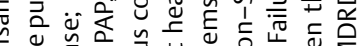

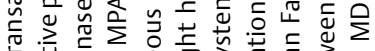

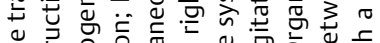

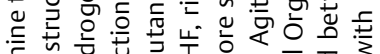

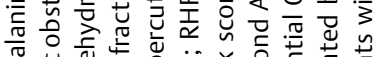

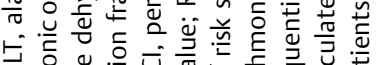

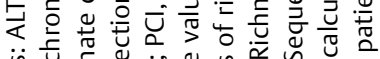

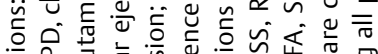
.0

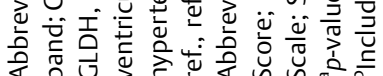


532 Thromboembolic and Bleeding Events in COVID-19 Patients Receiving ECMO Durak et al.

\section{Outcome and Clinical Course}

The median ECMO support in our 17 patients was 16 days (IQR: $10.5-22$ ). As of June 1, 2020, nine patients (53\%) have been weaned successfully from ECMO and survived to hospital discharge and eight patients (47\%) died during ECMO treatment. Seven patients (41\%) developed TEE and 10 patients (59\%) had BE during ECMO treatment. Patients frequently developed both TEE and BE; thus, five patients (71\%) of the TEE group had BE and five patients (50\%) of the BE group had TEE. Furthermore, we observed acute kidney failure with renal replacement therapy in 12 patients (71\%), bacterial superinfections in 8 patients (47\%), and right heart failure in 7 patients (41\%). More details can be found in - Table 2 .

Outcomes, usage of blood products, and hospitalization details between the investigated groups showed no statistically significant differences ( - Table 2). Nevertheless, some relevant differences could be found and are mentioned in this section.

Starting with the TEE group $(n=7)$ compared with the non-TEE group $(n=10)$, we found comparable values for mortality (43 vs. $50 \%, p=0.581$ ) and median ECMO duration with 16 days (IQR: 13-23) versus 14 days (IQR: 10-22), $p=0.601$. However, the TEE group developed right heart failure more frequently with 71 versus $20 \%(p=0.058)$ and the median ICU stay was longer with 37 days (IQR: 18-62) versus 23 days (IQR: $6-53), p=0.315$.

As for the BE group $(n=10)$, we found higher mortality rates than in the non-BE group $(n=7)$ with 60 versus $29 \%$ $(p=0.335)$ and more patients who developed a disseminated intravascular coagulation (DIC) with 40 versus $0 \%, p=0.103$. Usage of blood products during ECMO was higher in the BE

Table 2 Outcomes and clinical course

\begin{tabular}{|c|c|c|c|c|c|c|c|}
\hline Variable & Total $(n=17)$ & TEE $(n=7)$ & $\begin{array}{l}\text { No TEE } \\
(n=10)\end{array}$ & $p$-value ${ }^{a}$ & $\mathrm{BE}(n=10)$ & No $B E(n=7)$ & $p$-value ${ }^{a}$ \\
\hline Mortality, n (\%) & $8(47)$ & $3(43)$ & $5(50)$ & 0.581 & $6(60)$ & $2(29)$ & 0.335 \\
\hline TEE, $n(\%)$ & $7(41)$ & $7(100)$ & $0(0)$ & $0.000^{\mathrm{a}}$ & $5(50)$ & $2(29)$ & 0.622 \\
\hline $\mathrm{BE}, n(\%)$ & $10(59)$ & $5(71)$ & $5(50)$ & 0.622 & $10(100)$ & $0(0)$ & $0.000^{\mathrm{a}}$ \\
\hline Right heart failure, $n(\%)^{\mathrm{b}}$ & $7(41)$ & $5(71)$ & $2(20)$ & 0.058 & $4(40)$ & $3(43)$ & 1.000 \\
\hline Bacterial superinfection, $n(\%)$ & $8(47)$ & $2(29)$ & $6(60)$ & 0.335 & $4(40)$ & $4(57)$ & 0.637 \\
\hline Fungal superinfection, $n(\%)$ & $3(18)$ & $1(14)$ & $2(20)$ & 1.000 & $2(20)$ & $1(14)$ & 1.000 \\
\hline DIC, $n(\%)$ & $4(24)$ & $2(29)$ & $2(20)$ & 1.000 & $4(40)$ & $0(0)$ & 0.103 \\
\hline Delirium, $n(\%)$ & $4(24)$ & $1(14)$ & $3(30)$ & 0.603 & $2(20)$ & $2(29)$ & 1.000 \\
\hline Acute kidney failure, $n(\%)^{c}$ & $12(71)$ & $6(86)$ & $6(60)$ & 0.338 & $9(90)$ & $3(43)$ & 0.101 \\
\hline RRT initiated, $n(\%)$ & $12(71)$ & $6(86)$ & $6(60)$ & 0.338 & $9(90)$ & $3(43)$ & 0.101 \\
\hline \multicolumn{8}{|l|}{ Blood products } \\
\hline $\begin{array}{l}\text { ECMO flow }(\mathrm{L} / \mathrm{min}) \\
\text { median }(\mathrm{IQR})^{\mathrm{d}}\end{array}$ & $4(3.7-4.4)$ & $4(3.2-4.2)$ & $4.2(3.7-4.4)$ & 0.417 & $\begin{array}{l}4.2 \\
(3.4-4.4)\end{array}$ & $4(3.8-4.4)$ & 0.887 \\
\hline $\begin{array}{l}\text { Total PRBCs (units), } \\
\text { median (IQR) }\end{array}$ & $32(24-46)$ & $33(26-39)$ & $31(15-55.3)$ & 0.813 & $\begin{array}{l}32 \\
(25.5-43.5)\end{array}$ & $33(12-52)$ & 0.669 \\
\hline $\begin{array}{l}\text { PRBCs during ECMO (units), } \\
\text { median (IQR) }\end{array}$ & $28(3-69)$ & $26(16-35)$ & $24(14-48.5)$ & 1.000 & $\begin{array}{l}28 \\
(23.8-36.5)\end{array}$ & $14(8-35.8)$ & 0.093 \\
\hline $\begin{array}{l}\text { PRBC per ECMO (units/d), } \\
\text { median (IQR) }\end{array}$ & $\begin{array}{l}1.52 \\
(1.13-2.7)\end{array}$ & $\begin{array}{l}1.51 \\
(1.13-1.78)\end{array}$ & $\begin{array}{l}1.56 \\
(1.26-1.88)\end{array}$ & 0.669 & $\begin{array}{l}1.64 \\
(1.52-2.7)\end{array}$ & $\begin{array}{l}0.76 \\
(0.5-1.78)\end{array}$ & 0.270 \\
\hline $\begin{array}{l}\text { Total albumin (units), } \\
\text { median (IQR) }\end{array}$ & $85(4-266)$ & $9(1-251)$ & $103(9-300)$ & 0.669 & $124(8-365)$ & $12(0-167)$ & 0.230 \\
\hline \multicolumn{8}{|l|}{ Hospitalization details } \\
\hline Total LOS (d), median (IQR) & $24(11-56)$ & $37(14-64)$ & $23(6-41)$ & 0.417 & $20(6-56)$ & $30(14-65)$ & 0.417 \\
\hline $\begin{array}{l}\text { Total ICU stay (d), } \\
\text { median (IQR) }\end{array}$ & $24(16-56)$ & $37(18-62)$ & $23(6-53)$ & 0.315 & $30(17-58)$ & $24(1-53)$ & 0.669 \\
\hline $\begin{array}{l}\text { ECMO duration }(\mathrm{d}) \text {, } \\
\text { median (IQR) }\end{array}$ & $16(11-22)$ & $16(13-23)$ & $14(10-22)$ & 0.601 & $16(11-24)$ & $16(10-21)$ & 0.669 \\
\hline $\begin{array}{l}\text { iNO duration }(\mathrm{d}) \text {, } \\
\text { median (IQR) }\end{array}$ & $2(0-4.5)$ & $2(0-5)$ & $1.5(0-5.5)$ & 0.813 & $2.5(0.8-7)$ & $0(0-3)$ & 0.133 \\
\hline
\end{tabular}

Abbreviations: BE, bleeding events; DIC, disseminated intravascular coagulation; ECMO, extracorporeal membrane oxygenation; ICU, intensive care unit; iNO, inhaled nitric oxide; iQR, interquartile range; LOS, length of stay; MI, myocardial infarction; MV, mechanical ventilation; PRBCS, packed red blood cell; RRT, renal replacement therapy; TEE, thromboembolic events.

${ }^{a} p$-values are calculated between the two previous columns. Under 0.05 is considered significant.

' Including all patients with right heart failure in stage 3 or 4 by the American Heart Association guidelines.

'Including all patients with acute kidney failure in stage 2 or 3 by Kidney Disease Improving Global Outcomes (KDIGO) guidelines.

${ }^{\mathrm{d}}$ We presented the blood flow rate that was adjusted on the first day of ECMO therapy. 
Table 3 Thromboembolic and bleeding events (TEBE)

\begin{tabular}{|l|l|}
\hline Outcome variable & Total $(\boldsymbol{n}=\mathbf{1 7})$ \\
\hline Total TEBE, $\boldsymbol{n}(\%)$ & $12(71)$ \\
\hline TEE, $\boldsymbol{n}(\%)$ & $7(41)$ \\
\hline Pulmonary artery embolism & $5(29)$ \\
\hline Thrombosis & $3(18)$ \\
\hline arterial & $1(6)$ \\
\hline venous & $2(12)$ \\
\hline Ischemic or hemorrhagic stroke & $0(0)$ \\
\hline BE, $\boldsymbol{n}$ (\%) & $10(59)$ \\
\hline Upper respiratory tract & $8(47)$ \\
\hline endobronchial & $5(29)$ \\
\hline major & $2(12)$ \\
\hline minor & $3(18)$ \\
\hline mucosal & $7(41)$ \\
\hline major & $2(12)$ \\
\hline minor & $5(29)$ \\
\hline Pericardial tamponade & $2(12)$ \\
\hline Cannulation side & $5(29)$ \\
\hline major & $2(12)$ \\
\hline minor & $3(18)$ \\
\hline Gastrointestinal & $1(6)$ \\
\hline Hemothorax & $1(6)$ \\
\hline
\end{tabular}

Abbreviations: BE, bleeding events; TEE, thromboembolic events.

group; we also found more units of PRBC per ECMO day with 1.64 (IQR: $1.52-2.7$ ) versus 0.76 (IQR: $0.5-1.78$ ), $p=0.270$ and more units of albumin over the whole ICU stay with 124 (IQR: 8-365) versus 12 (IQR: $0-167$ ), $p=0.230$.

\section{Thromboembolic and Bleeding Events}

During ECMO therapy, we had 12 patients (71\%) who developed TEBE and 5 patients (29\%) had a clinical course without TEBE. We used anticoagulation with UFH in 16 patients (94\%). One patient $(6 \%)$ received the thrombin-inhibitor Argatroban instead of UFH, due to heparin-induced thrombocytopenia II (HIT II). This patient developed a PAE and a major endobronchial bleeding despite altered anticoagulation. Moreover, we found two patients $(12 \%)$ who already had TEBE after hospital admission but prior to ECMO implantation, and both had PAE. During ECMO, these two patients suffered again from PAE and developed bleedings.

- Table 3 shows the incidence of different TEBE during ECMO in detail. We summarized the results here. TEE occurred in 7 patients (41\%) and BE in 10 patients (59\%).

Among the seven patients with TEE were five patients (29\%) with a PAE. Three patients (18\%) had thrombosis consisting of two venous cases (12\%) and one arterial case (6\%). Both venous cases were a thrombosis in the right vena jugularis. The patient with arterial thrombosis had a thrombus formation in the fingers of the right hand. Ischemic or hemorrhagic strokes did not occur.
Among the 10 patients with BE were 8 patients (47\%) with a URT bleeding. To be precise, we observed seven patients (41\%) with a mucosal URT bleeding, of which two cases (12\%) were a major bleeding. Five patients (29\%) had an endobronchial URT bleeding, of which two cases $(12 \%)$ were major bleeding. There were five patients (29\%) with a cannulation side bleeding from which two cases $(12 \%)$ were a major bleeding. We also reported two patients (12\%) with a pericardial tamponade, one patient (6\%) with a GIB and one patient (6\%) with a hemothorax.

\section{Time Course of Coagulation Parameters}

Multiple parameters were measured on a daily basis to adjust anticoagulation therapy, where necessary. Due to imperfect documentation after ECMO initiation, we could not present all parameters. However, four different variables had less than $10 \%$ missing values and could be used. Therefore, we displayed time courses of PTT, platelet count, INR, and d-dimer using mean values in - Fig. 1.

PTT values were between 32 and 35 seconds before ECMO implantation and had an increasing trend during the therapy in all groups.

Regarding platelet count, the BE group had less platelets compared with the non-BE group in the first 3 days. As mentioned before, the difference in platelet count before ECMO implantation was statistically significant. However, the platelet count in the BE group was continuously above 80 $\mathrm{G} / \mathrm{L}$. This threshold was used in ECMO patients with bleeding by our internal guidelines.

INR values were higher in patients with TEE and BE. During statistical comparison, we could not find significant differences.

D-dimer values decreased in the first day after ECMO initiation; after that, we observed an increasing trend until the end of ECMO therapy in all patient groups.

\section{Discussion}

ARDS in COVID-19 patients showed a higher risk for TEBE than in non-COVID-19 patients. ${ }^{5}$ Considering the WHO and ELSO guidelines, ${ }^{10,18} \mathrm{ECMO}$ plays a role as a rescue therapy in case of critically ill COVID-19 patients suffering from hypoxemic respiratory failure. However, incidence and risk factors for TEBE in COVID-19 patients treated with ECMO are still uncertain.

In this study, we described TEBE in the first 17 critically ill COVID-19 patients who received ECMO in our center and reported the following key findings. TEBE occurred in 12 patients $(71 \%)$ consisting of 7 patients (41\%) with TEE and 10 patients (59\%) with BE. PAE was the most frequent TEE with five cases (29\%), and URT was the most common location for BE with eight cases (47\%). Mortality between the TEE group $(n=7)$ and the non-TEE group $(n=10)$ was comparable (43 vs. $50 \%, p=0.581)$, and higher in the BE group $(n=10)$ than in the non-BE group $(n=7)$, but without significance ( 60 vs. $29 \%$, $p=0.335$ ). From among 85 pre-ECMO variables, only platelet count was significantly lower between the BE group $(n=10)$ and the non-BE group ( $n=7)$ with 209 (IQR: $145-238)$ versus 452 (IQR: 240-560), $p=0.007$. However, we found other 

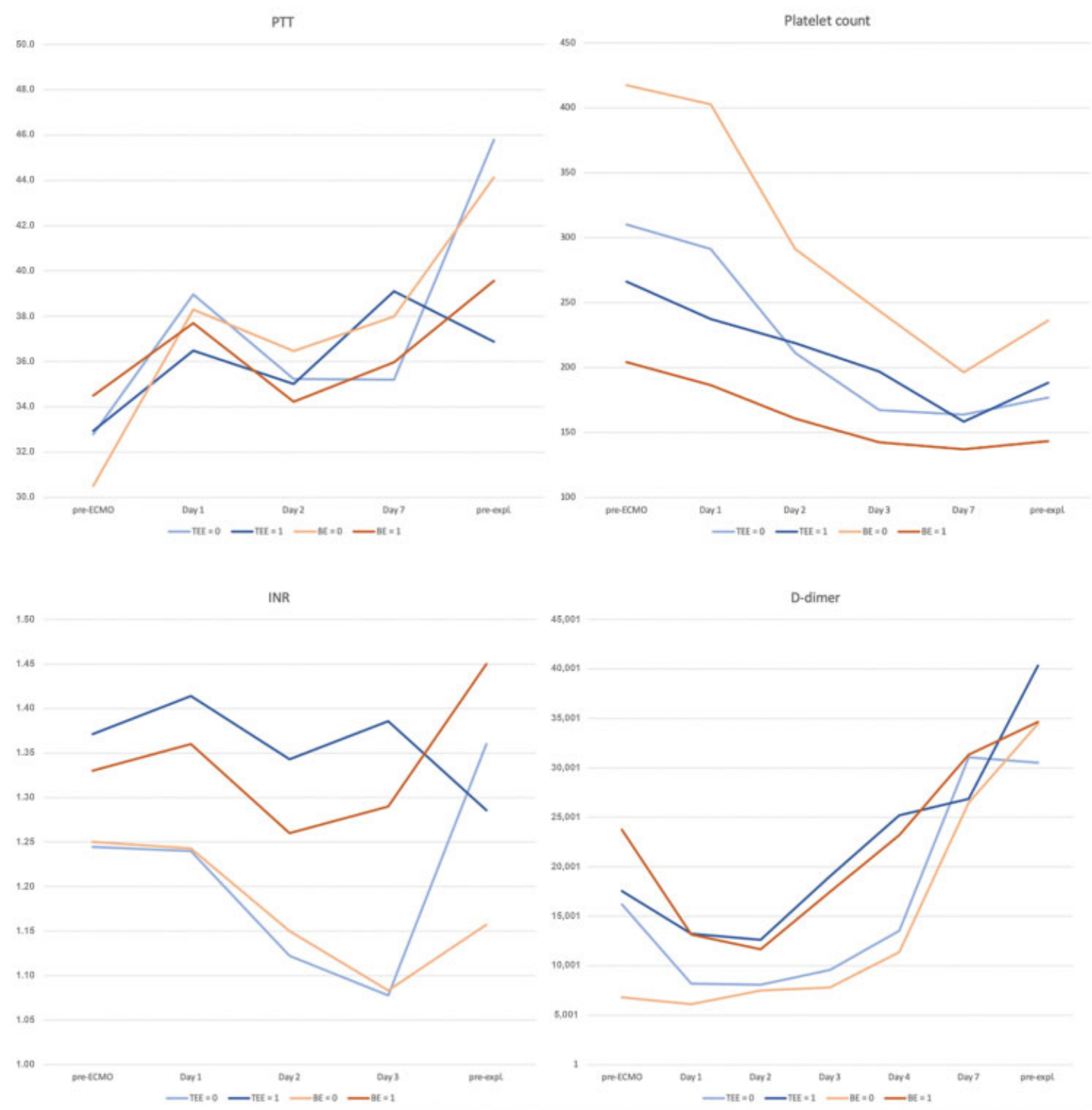

Fig. 1 Time courses of PTT, platelet count, INR, and d-dimer using mean values.

interesting differences that are not statistically significant but mentionable. TEE seemed to be more frequent patients with male gender, atrial fibrillation, less utilization of cytokine absorption, higher $\mathrm{pCO}_{2}$ values, and lower RESP score values. As for BE, we found longer PTT and lower INR values.

From among the TEBE, mainly BE were frequent. Regarding other studies about ECMO in patients suffering from severe ARDS without COVID-19, we also found a high incidence of BE. Two studies report BE in $46 \%$ of the patients; the first study included 124 patients and the second study had 214 patients. ${ }^{11,13}$ We compared it to the 10 patients (59\%) with BE of our study and no statistically significant differences could be found using Fisher's exact test. Only few studies reported the incidence of TEBE for COVID-19 patients treated with ECMO; the outcomes are not detailed enough and accurate comparisons with our study cannot be made. Especially the $\mathrm{BE}$ are more frequent in our study and this is probably due to the fact that we also reported minor bleedings. Nevertheless, we summarized the results of other studies in the following. Yang et $\mathrm{al}^{17}$ reported only 3 patients (14\%) with bleeding complications, whereas we had 10 patients (59\%). In an American study by Kon et al, ${ }^{20}$ there was only 1 patient (4\%) with clinically significant bleeding complications, 5 patients (19\%) with deep vein thrombosis (DVT), and 5 patients (19\%) who developed thrombocytopenia; ECMO-associated complications in general were noted in 11 patients (41\%). Bleeding complications and general ECMO complications are again much less than those in our study. However, we found four patients (24\%) with thrombosis as a comparable outcome. In the study of Jacobs et al, ${ }^{15}$ two patients (13\%) died due to DIC and one patient (7\%) died due to a cerebral bleeding. In another American study by Loforte et a ${ }^{21}$ with only four patients in 
total, one patient (25\%) suffered from GIB. In our study, we report also one patient (6\%) with GIB.

We observed a successful weaning rate of $53 \%$ from ECMO and survival to hospital discharge. This is superior to the rate of other studies. ${ }^{11,15,22}$ In the study of Yang et $\mathrm{al}^{23}$ only one patient (17\%) survived out of the six patients treated with ECMO.23 Marullo et $\mathrm{al}^{9}$ analyzed data of multiple institutions and reported a lower mortality of only $17 \%$ from 333 COVID-19 patients treated with ECMO. However, the actual weaning rate is $18 \%$ and the remaining patients are still alive on ECMO. ${ }^{9}$ Different patient selection and small sample sizes probably contribute to differences in survival rates.

The platelet count was significantly lower in patients with $\mathrm{BE}$ and therefore needs to be discussed. As stated previously, we only had one patient who developed a moderate thrombocytopenia between 50 and $100 \mathrm{G} / \mathrm{L}$. Nevertheless, thrombocytopenia is a common phenomenon found in ECMO patients and its underlying mechanisms are multifactorial. ${ }^{24}$ A decrease of platelets during the first 7 days is frequently seen and the values of our patient group were not clinically abnormal. ${ }^{24}$ We aimed for a platelet count above $80 \mathrm{G} / \mathrm{L}$ as recommended in the ELSO guidelines. ${ }^{25}$

We did not find any relevant hemorrhagic or ischemic strokes; however, we found five patients with microscopic bleedings in magnetic resonance imaging (MRI) examinations of the brain. Neurological symptoms did not occur in all cases and it is a common finding in COVID-19 patients without ECMO therapy. Therefore, it cannot be regarded as an ECMO complication in this study.

As a retrospective study, there was potential bias in data acquisition. Because of a small sample size and short study period, doctors could control the gathered data and confirm its correctness as much as possible. Although our pre-ECMO and outcome variables were complete, we have missing values for laboratory parameters during ECMO therapy. Therefore, we could not display time courses of all parameters.

We studied the characteristics and outcomes of the first 17 COVID-19 patients from March and April 2020, but we did not gather enough patients to conduct a high-quality predictor analysis. Therefore, validation of our findings with larger samples is required.

\section{Conclusion}

The present study describes TEBE during ECMO therapy in selected critically ill COVID-19 patients suffering from ARDS. It provides novel data about very common complications during ECMO therapy in a new subpopulation and can be used as guidance for future studies that investigate risk factors.

Further analysis with larger samples is required to optimize patient selection and improve the outcomes of critically ill COVID-19 patients.

\section{Authors' Contribution}

Koray Durak has collected the data (60\%), performed data

Analysis (55\%) and wrote the first version of the manu- script (100\%). Alex Kersten contributed to the Study design (50\%), performed ECMO implantation (35\%), collected the data (40\%), performed data analysis (45\%), supervised the writing process of the first version of the manuscript, critically revised the manuscript. The above mentioned contribution justify a contributed equally authorship of both authors Koray Durak and Alex Kersten.

\section{Acknowledgments}

We identified 10 different authors with the following explanation. All authors substantially contributed to the conception and performance of the study, as well as data acquisition, subsequent statistical analysis, and interpretation. Furthermore, multiple authors participated in ECMO implantation, blood sampling, supervision, and critical revision of the manuscript. All authors have seen and approved the final version of the manuscript. Both authors contributed equally to this study.

\section{Conflict of Interest}

None declared.

\section{References}

1 World Health Organization. Novel Coronavirus. Geneva: World Health Organization; 2020

2 Skevaki C, Fragkou PC, Cheng C, Xie M, Renz H. Laboratory characteristics of patients infected with the novel SARS-CoV-2 virus. J Infect 2020;81(02):205-212

3 Driggin E, Madhavan MV, Bikdeli B, et al. Cardiovascular considerations for patients, health care workers, and health systems during the covid-19 pandemic. J Am Coll Cardiol 2020;75(18): 2352-2371

4 Klok FA, Kruip MJHA, van der Meer NJM, et al. Incidence of thrombotic complications in critically ill ICU patients with COVID-19. Thromb Res 2020;191:145-147

5 Helms J, Tacquard C, Severac F, et al; CRICS TRIGGERSEP Group (Clinical Research in Intensive Care and Sepsis Trial Group for Global Evaluation and Research in Sepsis) High risk of thrombosis in patients with severe SARS-CoV-2 infection: a multicenter prospective cohort study. Intensive Care Med 2020;46(06): 1089-1098

6 Tang N, Li D, Wang X, Sun Z. Abnormal coagulation parameters are associated with poor prognosis in patients with novel coronavirus pneumonia. J Thromb Haemost 2020;18(04):844-847

7 Cui S, Chen S, Li X, Liu S, Wang F. Prevalence of venous thromboembolism in patients with severe novel coronavirus pneumonia. J Thromb Haemost 2020;18(06):1421-1424

8 Tang N, Bai H, Chen X, Gong J, Li D, Sun Z. Anticoagulant treatment is associated with decreased mortality in severe coronavirus disease 2019 patients with coagulopathy. J Thromb Haemost 2020;18(05):1094-1099

9 Marullo AG, Cavarretta E, Biondi Zoccai G, et al. Extracorporeal membrane oxygenation for critically ill patients with coronavirusassociated disease 2019: an updated perspective of the European experience. Minerva Cardioangiol 2020;68(05):368-372

10 World Health Organization. Clinical management of COVID-19: interim guidance. Geneva: World Health Organization; 2020

11 Combes A, Hajage D, Capellier G, et al; EOLIA Trial Group, REVA, and ECMONet. Extracorporeal membrane oxygenation for severe acute respiratory distress syndrome. N Engl J Med 2018;378(21): 1965-1975

12 Mendes PV, Melro LMG, Li HY, et al. Extracorporeal membrane oxygenation for severe acute respiratory distress syndrome in 
adult patients: a systematic review and meta-analysis. Rev Bras Ter Intensiva 2019;31(04):548-554

13 Munshi L, Walkey A, Goligher E, Pham T, Uleryk EM, Fan E. Venovenous extracorporeal membrane oxygenation for acute respiratory distress syndrome: a systematic review and metaanalysis. Lancet Respir Med 2019;7(02):163-172

14 Thomas J, Kostousov V, Teruya J. Bleeding and thrombotic complications in the use of extracorporeal membrane oxygenation. Semin Thromb Hemost 2018;44(01):20-29

15 Jacobs JP, Stammers AH, St Louis J, et al. Extracorporeal membrane oxygenation in the treatment of severe pulmonary and cardiac compromise in coronavirus disease 2019: experience with 32 patients. ASAIO j 2020;66(07):722-730

16 Kowalewski M, Fina D, Słomka A, et al. COVID-19 and ECMO: the interplay between coagulation and inflammation-a narrative review. Crit Care 2020;24(01):205

17 Yang X, Cai S, Luo Y, et al. Extracorporeal membrane oxygenation for coronavirus disease 2019-induced acute respiratory distress syndrome: a multicenter descriptive study. Crit Care Med 2020; 48(09):1289-1295

18 Bartlett RH, Ogino MT, Brodie D, et al. Initial ELSO guidance document: ECMO for COVID-19 patients with severe cardiopulmonary failure. ASAIO J 2020;66(05):472-474

19 Schulman S, Kearon CSubcommittee on Control of Anticoagulation of the Scientific and Standardization Committee of the
International Society on Thrombosis and Haemostasis. Definition of major bleeding in clinical investigations of antihemostatic medicinal products in non-surgical patients. J Thromb Haemost 2005;3(04):692-694

20 Kon ZN, Smith DE, Chang SH, et al. Extracorporeal membrane oxygenation support in severe COVID-19. Ann Thorac Surg 2020

21 Loforte A, Dal Checco E, Gliozzi G, et al. Veno-venous extracorporeal membrane oxygenation support in COVID-19 respiratory distress syndrome: initial experience. ASAIO J 2020;66(07):734-738

22 Peek GJ, Mugford M, Tiruvoipati R, et al; CESAR trial collaboration. Efficacy and economic assessment of conventional ventilatory support versus extracorporeal membrane oxygenation for severe adult respiratory failure (CESAR): a multicentre randomised controlled trial. Lancet 2009;374(9698):1351-1363

23 Yang X, Yu Y, Xu J, et al. Clinical course and outcomes of critically ill patients with SARS-CoV-2 pneumonia in Wuhan, China: a single-centered, retrospective, observational study. Lancet Respir Med 2020;8(05):475-481

24 Jiritano F, Serraino GF, Ten Cate H, et al. Platelets and extracorporeal membrane oxygenation in adult patients: a systematic review and meta-analysis. Intensive Care Med 2020;46(06): 1154-1169

25 Extracorporeal Life Support Organization. Guidelines for Adult Respiratory Failure. Ann Arbor, MI: Extracorporeal Life Support Organization; 2017 